\title{
Dynamics of interplanetary CMEs and associated type II bursts
}

\author{
Alejandro Lara ${ }^{1}$ and Andrea I. Borgazzi ${ }^{1,2}$ \\ ${ }^{1}$ Instituto de Geofísica \\ Universidad Nacional Autónoma de México (UNAM), México \\ email: alara@geofisisca.unam.mx \\ ${ }^{2}$ Divisão de Geofísica Espacial, INPE, Brasil \\ email: andrea@geofisica.unam.mx
}

\begin{abstract}
Coronal mass ejections (CMEs) are large scale structures of plasma $\left(\sim 10^{16} \mathrm{~g}\right)$ and magnetic field expelled from the solar corona to the interplanetary medium. During their travel in the inner heliosphere, these "interplanetary CMEs" (ICMEs), suffer acceleration due to the interaction with the ambient solar wind. Based on hydrodynamic theory, we have developed an analytical model for the ICME transport which reproduce well the observed deceleration of fast ICMEs. In this work we present the results of the model and its application to the CME observed on May 13, 2005 and the associated interplanetary type II burst.
\end{abstract}

Keywords. Sun: coronal mass ejections (CMEs), Sun: radio radiation, shock waves, solarterrestrial relations, solar wind

\section{Introduction}

The transport of interplanetary coronal mass ejections (ICMEs), has been studied for a few years, motivated mainly by the necessity of accurate Sun - Earth travel time predictions. The efforts to explain the ICME behavior may be divided into the following three categories (we include few examples in each case):

- Empirical models (Gopalswamy et al. 2000, 2001, 2005; Vršnak 2001; Vršnak et al. 2002, 2004, 2007).

- Numerical simulations (Cargill et al. 1996; Cargill 2004; Vandas et al. 1995; Odstrčil et al. 1999a,b; Gonzalez-Esparza et al. 2003).

- Theoretical models (Canto et al. 2005; Borgazzi et al. 2008).

Recently, we have developed an analytical method to explain the dynamics of ICMEs traveling in the ambient solar wind (SW). In this work we present two solutions of the model and the application of this model to the dynamics of the May 13, 2005 ICME and its associated Type II burst.

\section{The Model}

The forces acting on a body moving through a fluid are generically called "drag forces". Two kinds of drag forces are typically used, depending mainly on the velocity of the body, $U$ (in fact, the selection depends on the Reynolds number): the linear dependence, which we call "laminar", is $F_{l}=6 \pi \mu R \cdot U$, and the quadratic dependence, which we call "turbulent" is $F_{t}=\frac{C_{d} A \rho_{s w} \cdot U^{2}}{2}$, in this case, $\rho_{s w}$ is the interplanetary medium density; $R$ and $A$ are the ICME radius and cross section; $\mu$ is the viscosity and $C_{d}$ is the drag coefficient, a dimensionless parameter which describes the behavior of the body traveling through any fluid. 


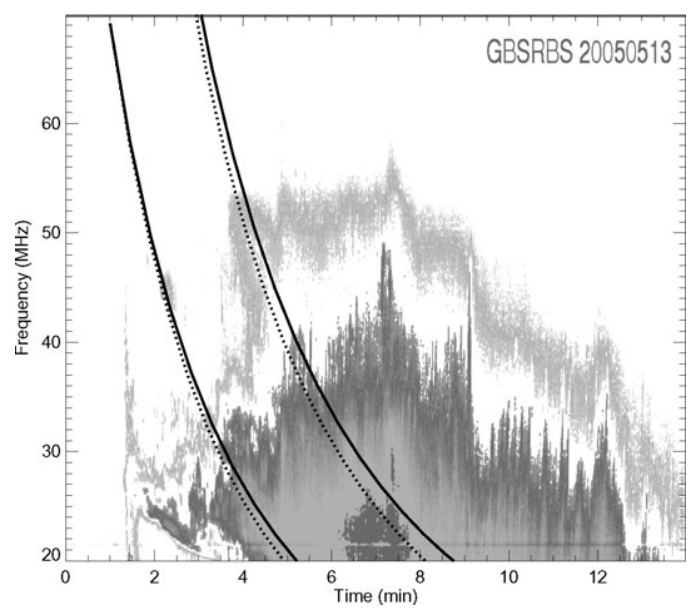

Figure 1. Spectrogram showing the metric type II burst, observed by GBSRBS, overplotted are the fundamental (lower curves) and harmonic (upper curves) solutions of our model. The starting time is $16: 41 \mathrm{UT}$.

Considering separately both forces in the equation of motion, and assuming variations of the ICME radius as $R(x)=x^{p}$ and SW density as $\rho_{s w}=x^{-2}$ (this approximation is valid from a few tens of $R_{\odot}$ to beyond 1 AU (Leblanc et al. 1996)), we have the following solutions:

$$
-\frac{6 \pi \nu \rho_{0}}{m_{c m e}(p-1)}\left[x^{p-1}-x_{0}^{p-1}\right]=U+U_{s w} \ln \frac{\left(U-U_{s w}\right)}{\left(U_{0}-U_{s w}\right)}-U_{0}
$$

and

$$
\begin{aligned}
- & \frac{C_{d} \pi \rho_{0}}{2 m_{c m e}(2 p-1)}\left[x^{(2 p-1)}-x_{0}^{(2 p-1)}\right] \\
& =\frac{U_{s w}}{\left(U_{0}-U_{s w}\right)}-\frac{U_{s w}}{\left(U-U_{s w}\right)}+\ln \left[\frac{\left(U-U_{s w}\right)}{\left(U_{0}-U_{s w}\right)}\right]
\end{aligned}
$$

for the laminar and turbulent cases, respectively. Here $m_{c m e}$ is the CME mass, $\rho_{0}$ is a scaling factor for the density model and corresponds to the density measured at $1 \mathrm{AU} ; U_{0}$ is the initial CME velocity measured at the initial position $x_{0} ; U_{S W}$ is the $\mathrm{SW}$ velocity; and $U$ is the ICME velocity at position $x$.

\section{Type II Bursts}

Type II bursts are produced when a disturbing agent is traveling, at relatively moderate speeds, through the solar atmosphere. This agent disturbs the ambient plasma which radiates electromagnetic emission at the plasma frequency. The agent may be a blast wave produced by a flare or a shock wave driven by a CME. At metric wavelengths, both mechanisms are plausible. At hectometric-decimetric and kilometric wavelengths, it is generally accepted that the agent is a shock wave produced by an ICME.

\subsection{The May 13, 2005 event}

At 17:22 UT on May 13, 2005 a very fast $\left(v_{c m e} \approx 1689 \mathrm{~km} / \mathrm{s}\right)$ halo CME was observed. This CME was associated with a M1.8 flare, localized close to the center of the disk (N12E11), starting at 16:13 UT, peaking at 16:57 UT, and ending at 17:28 UT. At coronal levels, a metric type II burst started at 16:42 UT at 30 and $60 \mathrm{MHz}$ for the fundamental and harmonic emissions, respectively (Figure 1). In the interplanetary medium, 


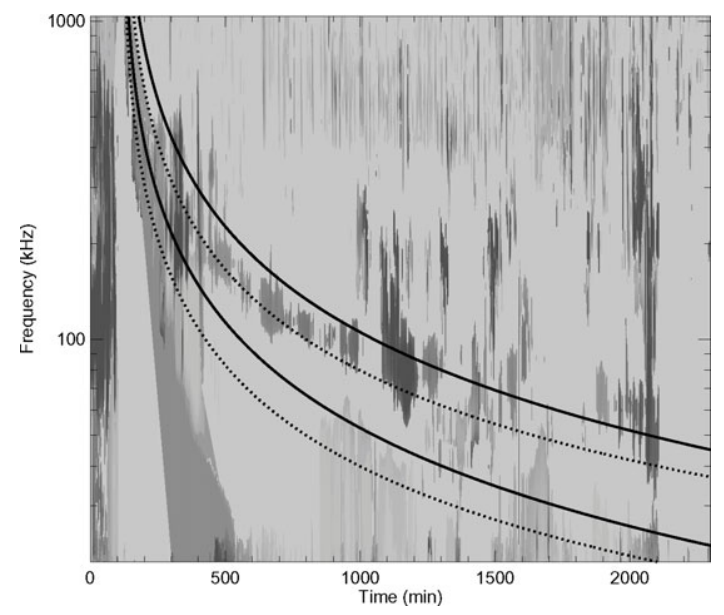

Figure 2. WIND/WAVES spectrogram showing the frequency evolution of the radio emission generated by the ICME driven shock. The starting time is 16:41 UT.

WIND/WAVES experiment detected an interplanetary type II burst, extending in frequency, from $20 \mathrm{kHz}$ to $13.825 \mathrm{MHz}$ and starting 16:00 UT on May 13, 2005 (Figure 2).

At the Earth, a sudden storm commencement (SSC) started at $\sim$ 02:38 UT on May 15 , indicating the arriving of the ejecta to $1 \mathrm{AU}$, followed by a strong (Dst $\approx-263 \mathrm{nT}$ ) geomagnetic storm. Therefore, the travel time, from May 13 17:22 (first LASCO/C2 observation) to May 15 02:38 (SSC) was $33 \mathrm{hr} 16 \mathrm{~min}$.

\section{Dynamics of the May 13, 2005 ICME}

In order to reproduce the ICME dynamics, indicated by the Type II bursts (which was produced by the ICME driven shock), we have used both, laminar (Eq. 2.1) and turbulent (Eq. 2.2) solutions and changed the parameters accordingly in order to fit both the computed ICME travel time $(\sim 33 \mathrm{hr})$ and the arriving velocity observed at 1 $\mathrm{AU}(\sim 1097 \mathrm{~km} / \mathrm{s})$ for this event. The following parameters correspond to the best fits and the correspondent solutions are over plotted in Figures 1 and 2:

- Laminar regime (solid lines):

$\circ U_{0}=1689 \mathrm{~km} / \mathrm{s}, p=0.77, \nu=1.03 \times 10^{21} \mathrm{~cm}^{2} / \mathrm{s}, m_{C M E}=10^{16} \mathrm{~g}$ and $U_{S W}=$ $415 \mathrm{~km} / \mathrm{s}$.

$\circ$ travel time $=33.8 \mathrm{hr}$. and ICME velocity at $1 \mathrm{AU}=1169.2 \mathrm{~km} / \mathrm{s}$

- Turbulent regime (dotted lines):

$\circ U_{0}=1689 \mathrm{~km} / \mathrm{s}, p=0.78, C_{d}=3.94 \times 10^{4}, m_{C M E}=10^{16} \mathrm{~g}$ and $U_{S W}=$ $415 \mathrm{~km} / \mathrm{s}$.

○ travel time $=33.0 \mathrm{hr}$. and ICME velocity at $1 \mathrm{AU}=1054.4 \mathrm{~km} / \mathrm{s}$

Equations 2.1 and 2.2 give the ICME instant velocity as a function of position. In order to have the ICME position as a function of time, we have divided the interval (1 AU) in 4092 equally spaced sectors, computed the mean velocity in each sector and then, computed the time assuming constant velocity in the sector. Once we had the ICME position as a function of time, we were able to obtain the density by assuming a SW density model $\left(n \sim 1 / x^{2}\right)$ and therefore, the plasma frequency $(f \sim \sqrt{n})$ as a function of time.

In this way, we can compare our results against the observed Type II behavior. In Figures 1 and 2, we have plotted the computed laminar (continuous line) and turbulent 
(dotted line) frequency drift. The lower pair of curves represent the fundamental and the upper pair represent the harmonic emissions. We selected the ICME starting time equal to the metric Type II burst starting time.

\section{Discussion - Conclusion}

The very good fits of both the coronal (Fig. 1) and interplanetary medium (Fig. 2) type II bursts with our model shows that the dynamics in the interplanetary medium of CMEs can be explained by the hydrodynamic theory. This means that the drag force plays a major role in the ICME transport, both linear or quadratic speed dependence are plausible. We note that in this case, and as we are dealing with low density collisionless plasmas, the viscosity and/or drag interactions should be produced by microscopic wave-wave or wave-particle interactions.

At large scales $(\sim 1 \mathrm{AU})$ there are not significant difference between the linear and quadratic dependence of the drag force with the ICME speed. Although, the quadratic model seems to fit better the curvature of the type II frequency drift, the travel time and the ICME velocity at $1 \mathrm{AU}$.

It is important to note that our approximation has few assumptions, as the radial expansion of the $\operatorname{ICME}\left(R_{I C M E}(x)=x^{p}\right)$ and the density decrease $\left(\rho(x)=\rho_{0} / x^{2}\right)$. The only free parameters are $C_{d}$ or $\nu$. This fact shows the advantage of using analytical models.

Finally, we quote the values of the drag coefficient, $C_{d} \approx 4 \times 10^{4}$ and kinematic viscosity, $\nu \approx 10^{21}$ which seems to reproduce well this event.

\section{Acknowledgements}

A. Lara thanks UNAM-PAPIT (IN117309) and CONACyT (49395). A. Borgazzi thanks CNPq-Brazil.

\section{References}

Borgazzi, A., Lara, A., Romero-Salazar, L., \& Ventura, A. 2008, Geofísica Internacional, 47, 301

Canto, J., Gonzalez, R. F., Raga, A. C., de Gouveia Dal Pino, E. M., Lara, A., \& GonzalezEsparza, A. 2005, Mon. Not R. Astrom. Soc., 357, 572

Cargill, P. J. \& Chen, J. 1996, J. Geophys. Res., 101, A3, 4855

Cargill, P. J. 2004, Sol. Phys., 221, 135

Gonzalez-Esparza, A., Lara, A., Perez-Tijerina, E., Santillan, A., \& Gopalswamy, N. 2003, J. Geophys. Res., 108, A1, 1039

Gopalswamy, N., Lara, A., Yashiro S., Kaiser, M. L., \& Russell H. 2001, J. Geophys. Res., 18, 29.207

Gopalswamy, N., Lara, A., Lepping, R. P., Kaiser, M. L., Berdichevsky, D., \& St,. Cyr, O. C. 2000, Geophys. Res. Lett., 27, 145

Gopalswamy, N., Lara, A., Manoharan, P., \& Howard, R. 2005, Adv. Space Res., 36, 2289

Leblanc, Y., Dulk, G., \& Bougeret, J. 1996, Solar Phys., 183, 165

Odstrčil, D. \& Pizzo, V. J. 1999a, J. Geophys. Res., 104, A1, 483

Odstrčil, D. \& Pizzo, V. J. 1999b, J. Geophys. Res., 104, A1, 493

Vandas, M., Fisher, S., Dryer, M., Smith, M., \& Detman, T. 1995, J. Geophys. Res., 100,A7, 12258

Vršnak, B. 2001, J. Geophys. Res., 106, A11, 25249

Vršnak, B. \& Gopalswamy, N. 2002, J. Geophys. Res., 107, A2

Vršnak, B., Ruždjak, D., Sudar, D., \& Gopalswamy, N. 2004, A\&̊A, 423, 717

Vršnak, B. \& Žic, T. 2007, A\&̈A, 472, 937 